\title{
One-dimensional photonic crystal with a complex defect containing an ultrathin superconducting sublayer
}

\author{
N. N. Dadoenkova, ${ }^{1, a), e)}$ A. E. Zabolotin, ${ }^{1}$ I. L. Lyubchanskii, ${ }^{1, b), e)}$ Y. P. Lee, ${ }^{2, c)}$ and \\ Th. Rasing ${ }^{3, d)}$ \\ ${ }^{1}$ Donetsk Physical and Technical Institute, National Academy of Sciences of Ukraine, \\ 83114 Donetsk, Ukraine \\ ${ }^{2}$ Department of Physics and Quantum Photonic Science Research Center ( $q$-Psi), Hanyang University, \\ Seoul 133-791, South Korea \\ ${ }^{3}$ Radboud University of Nijmegen, Institute for Molecules and Materials, Heyendaalseweg 135, \\ 6525 AJ Nijmegen, The Netherlands
}

(Received 23 June 2010; accepted 29 August 2010; published online 11 November 2010)

\begin{abstract}
The influence of the variation in the incidence angle on the photonic band gap spectra of a one-dimensional dielectric photonic crystal with a complex defect layer, consisting of ultrathin superconducting and dielectric sublayers, was theoretically investigated. The behavior of the defect modes with different polarizations as a function of the incidence angle variation is studied numerically for different thicknesses of the superconducting sublayer. The pronounced contrast in behavior of TE- and TM-polarized modes was demonstrated. The intensity of the TE-polarized defect mode decreases with increasing incidence angle, whereas the intensity of the TM-polarized defect mode increases. The increase in the superconducting defect sublayer thickness leads to a shift in the defect mode of TM-polarization to higher frequencies. (c) 2010 American Institute of Physics.
\end{abstract} [doi:10.1063/1.3494034]

\section{INTRODUCTION}

During the last two decades much attention has been focused on the theoretical and experimental investigation of photonic crystals (PCs) or photonic band gap (PBG) materials, due to promising applications of PCs in modern photonics. ${ }^{1,2}$ These PCs can be presented as periodic onedimensional (1D), two-dimensional (2D), or threedimensional structures, composed at least of two different materials with periods, comparable to the wavelength of the incident electromagnetic wave (EMW). The difference in the refractive indices (RIs) values of the PC's components leads to the appearance of so-called PBGs in the spectra of the normal EMWs, i.e., to forbidden regimes where EMWs cannot propagate through the PC. It is well known that introduction of an extra element into the regular photonic structure destroys the periodicity and leads to the appearance of a narrow peak with high transmittivity at a frequency inside the PBG, the so-called defect mode. ${ }^{1-3}$

For a long time intense efforts were devoted to studying PCs composed of conventional materials: dielectrics and metals. Nevertheless, such unconventional constituents for PCs as magnetic and ferroelectric materials were investigated as well. ${ }^{4-14}$ Another group of unconventional materials for PCs, namely, superconductors was also studied in a number of theoretical publications for 1D and 2D PCs. ${ }^{15-33}$ All the articles related to superconducting (SC) PCs were inves-

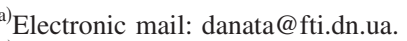

${ }^{b)}$ Electronic mail: igorl@fti.dn.ua.

${ }^{c)}$ Electronic mail: yplee@ hanyang.ac.kr.

${ }^{d)}$ Electronic mail: th.rasing @ science.ru.nl.

e) Also at: Department of Physics, Donetsk National University, Donetsk 83000, Ukraine.
}

tigating regular (nondefect) structures. The interest to the SC $\mathrm{PCs}$ is due to their applications in a new type of resonators ${ }^{34}$ and microstrip filters based on $\mathrm{YBa}_{2} \mathrm{Cu}_{3} \mathrm{O}_{7}$ which work in the gigahertz regime. ${ }^{35}$ By tuning the temperature of the superconductor, the RI of the SC component can be changed, and as a consequence, the PBG can be controlled. Quite recently a tunable Mach-Zehnder interferometer with a 2D photonic structure composed of copper oxide hightemperature superconductor has been proposed in Ref. 36. In this paper ${ }^{36}$ large tunability of the PBG in the temperature interval from $5 \mathrm{~K}$ till $105 \mathrm{~K}$ in the SC state is demonstrated. The SC PCs based devices potentially can find application in high frequency electromagnetic circuits from microwave until optical regimes [including terahertz $(\mathrm{THz})$ frequencies], which is very important for lossless communication systems and networks working at liquid nitrogen temperatures.

In a recent paper, ${ }^{37}$ the influence of a thin SC layer on the transmission, reflection, and absorption in a 1D dielectric PC was theoretically studied. As shown in Ref. 37, a thin SC layer, deposited on one side of a dielectric PC, leads to a decrease in the transmittivity for an EMW polarized along the $x$-axis, whereas a $y$-polarized EMW propagates through this PC practically without losses.

In our recent paper ${ }^{38}$ we presented the results of a theoretical investigation of the light transmission through a 1D dielectric PC with a complex defect layer composed of SC and dielectric parts. The influence of a SC sublayer with different thicknesses on the behavior of the defect mode inside the PBG was studied at different temperatures. It was shown that the position of the defect mode and the value of the transmittivity at the defect mode frequency strongly depend on the thickness of the SC sublayer, as well as on the temperature. In that paper $^{38}$ we investigated the case of nor- 


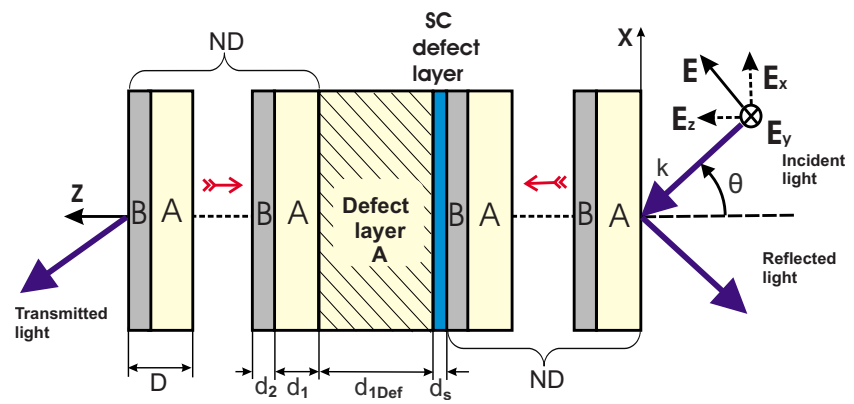

FIG. 1. (Color online) Schematic of the finite two-component 1D PC $(A B)^{5} A_{d e f} S C(A B)^{5}$ with the combined SC defect layer.

mal incidence of the EMW only. Nevertheless, the effects coming from an oblique incidence of EMWs with different polarizations are important, as theoretically demonstrated in Ref. 28 for the regular superconductor-dielectric PC.

In this paper we extend our investigation on the transmittivity, reflectivity, and absorptance spectra of a PC with a complex SC defect in the case of oblique incidence of light.

The paper is organized as follows. In Sec. II, we describe the model and analytical approach. In Sec. III the numerical calculation of the transmittivity, reflectivity, and absorption spectra for a 1D PC, composed of dispersionless strontium titanate and aluminum oxide, and a defect layer, composed of a dispersive $\mathrm{SC}$ sublayer of $\mathrm{YBa}_{2} \mathrm{Cu}_{3} \mathrm{O}_{7}$ and a sublayer of strontium titanate, are presented. In the Conclusions we summarize the obtained results.

\section{MODEL AND METHOD}

Let us consider a finite size 1D PC of the structure $(B A)^{N} \operatorname{Def}(B A)^{N}$ consisting of two regular PCs $(B A)^{N}$ and the combined defect layer $D e f=A_{d e f} \mathrm{SC}$ embedded between them, as depicted in Fig. 1. The combined defect consists of the SC sublayer of thickness $d_{s}$ and the dielectric sublayer A of thickness $d_{1 D e f}$, respectively. We assume the layers A of thickness $d_{1}$ to be strontium titanate $\mathrm{SrTiO}_{3}$. The layers $\mathrm{B}$ of thickness $d_{2}$ are aluminum oxide $\mathrm{Al}_{2} \mathrm{O}_{3}$. The $\mathrm{SC}$ sublayer we consider to be $\mathrm{YBa}_{2} \mathrm{Cu}_{3} \mathrm{O}_{7}$. The period of the regular structure is $D=d_{1}+d_{2}$. The selection of these dielectrics is due to the fact that both these materials $\mathrm{SrTiO}_{3}$ and $\mathrm{Al}_{2} \mathrm{O}_{3}$ are widely used as substrates for $\mathrm{YBa}_{2} \mathrm{Cu}_{3} \mathrm{O}_{7} \mathrm{SC}$ films. The medium surrounding the PC is vacuum. The PC's layers are located in the $x y$-plane and the $z$-axis is perpendicular to the interfaces.

We consider the case of oblique incidence of the light on the right hand surface of the PC under the incidence angle $\theta$ (see Fig. 1). The incident light is linearly polarized: TE- or TM-polarizations (the EMWs with electric field vector $\mathbf{E}$ along $y$-axis or in $x z$-plane, respectively).

The transmittivity and reflectivity spectra of the 1D PC can be calculated by means of a $(4 \times 4)$ transfer matrix method which is introduced by Berreman ${ }^{39}$ and successfully used by $\mathrm{Yeh}^{40,41}$ for anisotropic 1D periodic structures and by us for 1D magneto-optical PCs. ${ }^{42}$ The transfer matrix $\hat{T}$ for the PC under consideration can be presented as follows:

$$
\hat{T}=\hat{S}_{01}\left(\hat{T}_{0}\right)^{5} \hat{S}_{d e f}\left(\hat{T}_{0}\right)^{5} \hat{S}_{10},
$$

where the matrix $\hat{S}_{10}$ describes the EMW transition through the boundary "vacuum-medium A" and $\hat{T}_{0}$ is a transfer matrix for the unit cell of the PC

$$
\hat{T}_{0}=\hat{S}_{12} \hat{E}_{2}\left(d_{2}\right) \hat{S}_{21} \hat{E}_{1}\left(d_{1}\right),
$$

defined as product of the matrices $\hat{S}_{12}$ and $\hat{S}_{21}\left(\hat{S}_{12}=\hat{S}_{21}^{-1}\right)$, connecting the EMW's field amplitudes at the different sides of the boundaries "medium A-medium B." They are defined as

$$
\hat{S}_{10}=\left(\hat{A}_{1}\right)^{-1} \hat{A}_{0},
$$

with $(4 \times 4)$ matrixes $\hat{A}_{j}(j=0,1,2)$ written in the quasidiagonal form

$$
\hat{A}_{j}=\left(\begin{array}{cc}
\hat{A}_{j}^{(\mathrm{TE})} & \hat{O} \\
\hat{O} & \hat{A}_{j}^{(\mathrm{TM})}
\end{array}\right),
$$

where $\hat{O}$ denotes a $(2 \times 2)$ zero matrix and $\hat{A}_{j}^{(\mathrm{TE})}$ and $\hat{A}_{j}^{(\mathrm{TM})}$ describe the EMW's amplitude coefficients for the TE- and TM-modes

$$
\hat{A}_{j}^{(\mathrm{TE})}=\left(\begin{array}{cc}
1 & 1 \\
\delta_{\epsilon, j} & -\delta_{\epsilon, j}
\end{array}\right), \quad \hat{A}_{j}^{(\mathrm{TM})}=\left(\begin{array}{cc}
1 & 1 \\
-\delta_{\mu, j} & \delta_{\mu, j}
\end{array}\right),
$$

where the matrix components are determined as

$$
\delta_{\epsilon, j}=k_{j z} c /\left(\omega \mu_{j}\right), \quad \delta_{\mu, j}=k_{j z} c /\left(\omega \epsilon_{j}\right),
$$

with the wave vector $z$-components

$$
k_{j z}=\sqrt{-k_{x}^{2}+(\omega / c)^{2} \epsilon_{j} \mu_{j}} .
$$

The diagonal matrices $\hat{E}_{j}\left(d_{j}\right), j=1,2$ in Eq. (2) present the phase incursions within the corresponding layers

$$
\hat{E}_{j}\left(d_{j}\right)=\operatorname{diag}\left(e^{i k_{j z} d_{j}}, e^{-i k_{j z} d_{j}}, e^{i k_{j z} d_{j}}, e^{-i k_{j z} d_{j}}\right) .
$$

In Eq. (1) the defect layer matrix $\hat{S}_{\text {def }}$ can be written as

$$
\hat{S}_{d e f}=\hat{E}_{d 1}\left(d_{1 D e f}\right) \hat{S}_{1 s} \hat{E}_{s}\left(d_{s}\right) \hat{S}_{s 1},
$$

where $\hat{S}_{s 1}$ connects the field amplitudes at the boundaries of the medium A and the SC sublayer, labeled as " $s$ "

$$
\hat{S}_{1 s}=\left(\hat{A}_{1}\right)^{-1} \hat{A}_{s} \text {. }
$$

The matrix $\hat{A}_{s}$ has a form similar to $\hat{A}_{j}$, and is defined by Eqs. (4) and (5) with

$$
\delta_{\epsilon, s}=k_{s}^{(\mathrm{TE})} c /\left(\omega \mu_{s}\right), \quad \delta_{\mu, s}=k_{s}^{(\mathrm{TM})} c /\left[\omega \epsilon_{x, s}(\omega)\right],
$$

where $k_{s z}^{(\mathrm{TE})}$ and $k_{s z}^{(\mathrm{TM})}$ are the $z$-components of the EMWs of TE- and TM-polarizations in the SC sublayer

$$
\begin{aligned}
& k_{s z}^{(\mathrm{TE})}=\sqrt{-k_{x}^{2}+(\omega / c)^{2} \epsilon_{s, y}(\omega) \mu_{s}}, \\
& k_{s z}^{(\mathrm{TM})}=\sqrt{-k_{x}^{2}+(\omega / c)^{2} \epsilon_{s, x}(\omega) \mu_{s} .}
\end{aligned}
$$

Obviously, the phase incursion matrices $\hat{E}_{s}\left(d_{s}\right)$ and $\hat{E}_{d 1}\left(d_{1 D e f}\right)$ in Eq. (9) refer to the SC and dielectric parts of the combined defect layer 
(a)
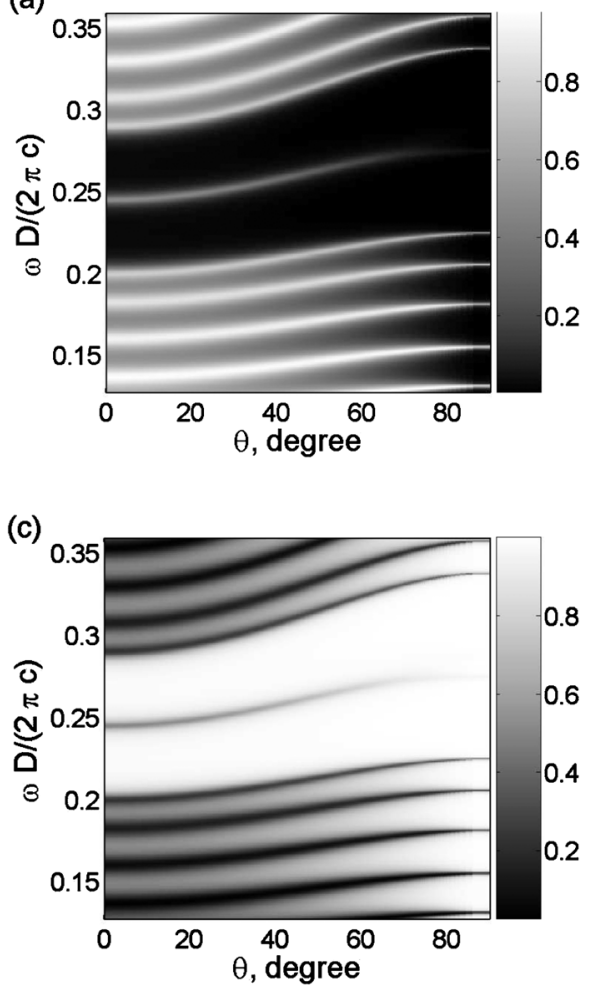

(b)

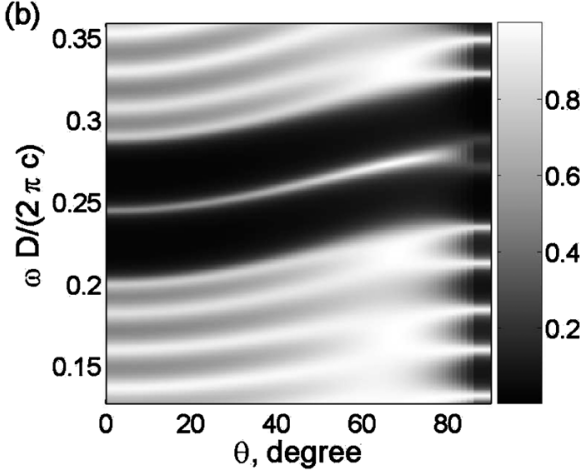

(d)

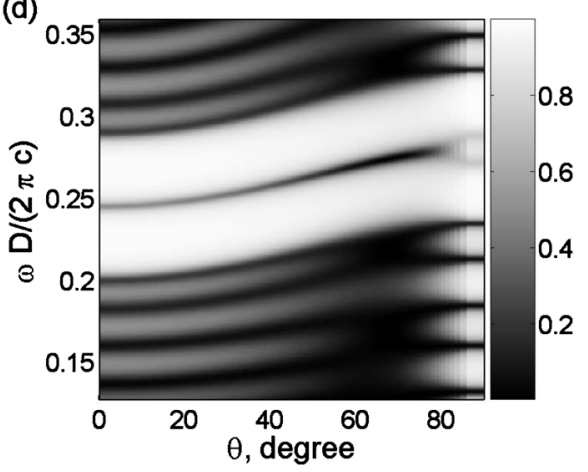

FIG. 2. The top view of the transmittivities $T^{(\mathrm{TE}, \mathrm{TE})}$ (a), $T^{(\mathrm{TM}, \mathrm{TM})}$ (b), and reflectivities $R^{\text {(TE,TE) }}(\mathrm{c})$ and $R^{\text {(TM,TM) }}$ (d) vs the normalized frequency $\omega D /(2 \pi c)$ (in the range of the first PBG and its vicinity) and the incidence angle $\theta$. The results are obtained for the case of the dielectric defect layer $d_{1 d e f}=d_{1}$ without the $\mathrm{SC}$ sublayer.

$$
\begin{aligned}
& \hat{E}_{s}\left(d_{s}\right)=\operatorname{diag}\left(e^{i k_{s z}^{(\mathrm{TE})} d_{s}}, e^{-i k_{s z}^{(\mathrm{TE})} d_{s}}, e^{i k_{s z}^{(\mathrm{TM})} d_{s}}, e^{-i k_{s z}^{(\mathrm{TM})} d_{s}}\right) \\
& \hat{E}_{d 1}\left(d_{1 D e f}\right)=\operatorname{diag}\left(e^{i k_{1 z} d_{1 D e f}}, e^{-i k_{1 z} d_{1 D e f}}, e^{i k_{1 z} d_{1 D e f}}, e^{-i k_{1 z} d_{1 D e f}}\right) .
\end{aligned}
$$

In our consideration we used the frequency- and temperature-dependent dielectric permittivity tensor with nonzero diagonal components $\epsilon_{x x}(\omega, T)$ and $\epsilon_{y y}(\omega, T)$ $=\epsilon_{z z}(\omega, T)$, introduced in Ref. 37

$$
\epsilon_{s, \nu}(\omega, T)=1+\frac{4 \pi i}{\omega} \sigma_{s, \nu}(\omega, T), \quad \nu=x x, y y,
$$

Here, the dynamic electrical conductivity tensor $\sigma_{s, \nu}(\omega, T)$ is defined as

$$
\sigma_{s, \nu}(\omega, T)=f_{+}(\omega, T) \sigma_{s, \nu}^{+}(\omega, T)+f_{-}(\omega, T) \sigma_{s, \nu}^{-}(\omega, T),
$$

where $\sigma_{s, \nu}^{ \pm}(\omega, T)$ are determined as follows:

$$
\begin{aligned}
& \sigma_{s, \nu}^{+}(\omega, T)=\frac{i\left(T^{4}-T_{c}^{4}\right)}{\omega \Lambda_{s, \nu}^{(0)} T_{c}^{4}}+\left(\frac{T}{T_{c}}\right)^{4} \sigma_{s, \nu}^{-}(\omega, T), \\
& \sigma_{s, \nu}^{-}(\omega, T)=\frac{T_{c}}{\Lambda_{s, \nu}^{(0)}\left(\alpha T-i \omega T_{c}\right)},
\end{aligned}
$$

where $T_{c}$ and $f_{ \pm}(\omega, T)$ are critical temperature and the electron distribution functions, respectively,

$$
f_{ \pm}(\omega, T)=\left(\exp \left\{\frac{ \pm \hbar\left[\omega-\omega_{s}(T)\right]}{k_{B} T}\right\}+1\right)^{-1} .
$$

In Eq. (16) $\omega_{s}$ is determined via the temperature-dependent superconductor half-gap energy $\Delta(T)$ as $\hbar \omega_{s}=\Delta(T)$, which is approximated as $\Delta(T) \cong \Delta_{0}\left[1-\left(T^{3} / T_{c}^{3}\right)\right]^{1 / 4}$ (see for details Ref. 37).

\section{NUMERICAL RESULTS AND DISCUSSION}

In this section, we analyze numerically both transmittivity and absorption spectra as function of the incidence angle $\theta$ between $0^{\circ}$ and $90^{\circ}$ for a PC with the SC sublayer thickness $d_{s}$ fixed to be 10,20, and $30 \mathrm{~nm}$. In the present paper we restrict our consideration to spectra with the first (lowest in frequency) PBG. As in our previous paper, ${ }^{38}$ for the numerical calculations presented below we chose the following parameters of the PC: the unit cell number $N=5$, the PC's pe$\operatorname{riod} D=5 \mu \mathrm{m}$, and the dielectric layer thicknesses $d_{1}$ $=0.42 \mathrm{D}$ and $d_{2}=0.58 \mathrm{D}$. The thickness of the dielectric defect sublayer is equal to $d_{1 D e f}=0.42 D=2.1 \mu \mathrm{m}$. We calculated the optical properties of the PC at the temperature $T=77 \mathrm{~K}$ (below $T_{c}$ ). The RI's for the PC's constituents are equal to $n_{1}=2.437\left(\right.$ for $\left.\mathrm{SrTiO}_{3}\right), n_{2}=1.767\left(\right.$ for $\left.\mathrm{Al}_{2} \mathrm{O}_{3}\right){ }^{43}$

It should be noted that in the optical and near infrared ranges the electrodynamic properties of $\mathrm{YBa}_{2} \mathrm{Cu}_{3} \mathrm{O}_{7}$ can be described by a dielectric permittivity only, i.e., the magnetic permeability is assumed to be $\mu_{s}=1$.

For an estimation of the frequency and temperature dependence of $\varepsilon_{s, \nu}$ [see Eq. (14)] we used the following parameters: the scattering rate component $\alpha=1.4 \times 10^{13} \mathrm{~s}^{-1}$, the London tensor components at absolute zero $\Lambda_{s, x}^{(0)}=2.1$ $\times 10^{-30} \mathrm{~s}^{2}$ and $\Lambda_{s, y}^{(0)}=1.9 \times 10^{-28} \mathrm{~s}^{2}$, as well as the half-gap energy at absolute zero $\Delta_{0}=3.0 \times 10^{-2} \mathrm{eV}^{37}$ The critical temperature for $\mathrm{YBa}_{2} \mathrm{Cu}_{3} \mathrm{O}_{7}$ is $T_{c}=90 \mathrm{~K}$.

First, we start our consideration with a PC having a pure dielectric defect (when the SC sublayer is missing, $d_{s}=0$ ). In Figs. 2(a) and 2(b) the transmittivities for EMW's of TE- and TM-polarizations $T^{(\mathrm{TE}, \mathrm{TE})}$ and $T^{(\mathrm{TM}, \mathrm{TM})}$ are given versus the incidence angle $\theta$ and normalized frequency $\omega D /(2 \pi c)$ within the first PBG and its vicinity. For the considered structure the incident light of TE- (or TM-) polarization pro- 

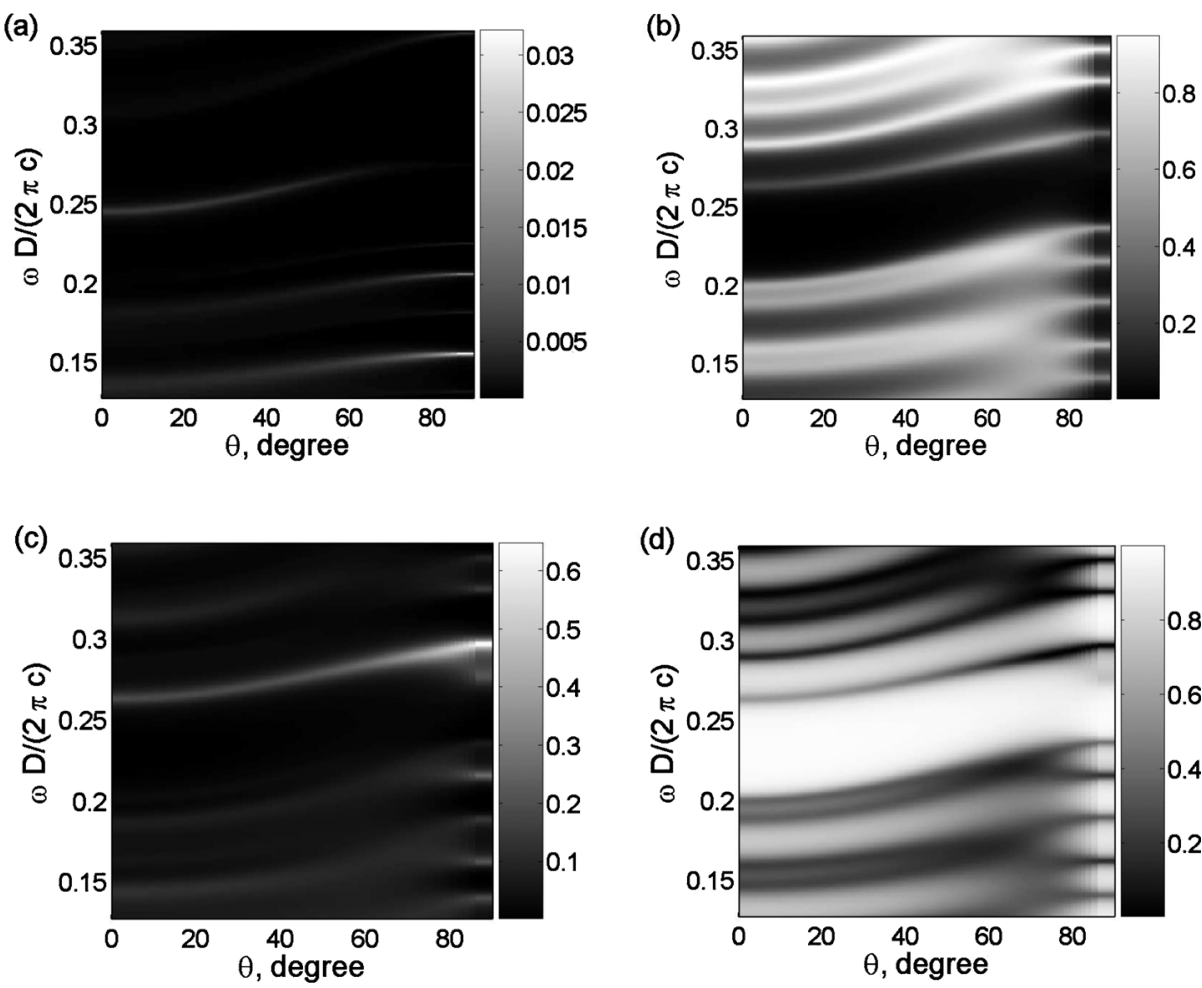

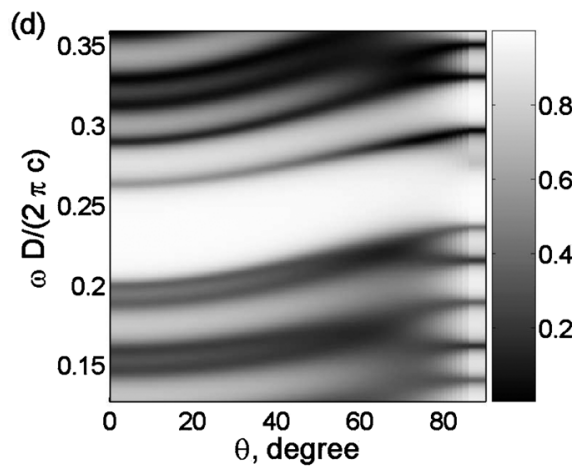

FIG. 3. The top view of the absorptance $A^{\text {(TE,TE) }}$ (a) and $A^{\text {(TM,TM) }}$ (c), transmittivity $T^{(\mathrm{TM}, \mathrm{TM})}(\mathrm{b})$, and reflectivity $R^{\text {(TM,TM) }}$ (d), vs the normalized frequency $\omega D /(2 \pi c)$ and the incidence angle $\theta$. The calculations are performed for the $\mathrm{PC}$ with the dielectric and SC defect layers of thicknesses $d_{1 d e f}=d_{1}$ and $d_{s}=10 \mathrm{~nm}$, respectively, for $T=77 \mathrm{~K}$ duces the reflected wave of the same polarization: TE (or $\mathrm{TM})$. In other words the off-diagonal components of the reflection and transmission matrixes $T^{(\mathrm{TE}, \mathrm{TM})}$ and $T^{(\mathrm{TM}, \mathrm{TE})}$ are zeros.

The corresponding reflectivities $R^{(\mathrm{TE}, \mathrm{TE})}$ and $R^{(\mathrm{TM}, \mathrm{TM})}$ for TE- and TM-modes are depicted in Figs. 2(c) and 2(d).

In the considered frequency range both dielectrics $\mathrm{SrTiO}_{3}$ and $\mathrm{Al}_{2} \mathrm{O}_{3}$ are not absorbing media, so the absorbtion in the PC of the structure $(B A)^{N} A_{d e f} \mathrm{SC}(B A)^{N}$ is especially due to presence of the SC defect sublayer. In the absence of the SC insert, the energy conservation law leads to the obvious relation $T^{(\gamma)}+R^{(\gamma)}=1,{ }^{44}$ where $\gamma=\mathrm{TE}, \mathrm{TM}$ denotes the polarization of the EMW. So, for corresponding polarizations the transmittivity spectra in Figs. 2(a) and 2(c) and the reflectivity spectra in Figs. 2(b) and 2(d) are mutually complementary.

In Figs. 2(a)-2(d) one can see, that for both TE- and TM-modes the increase in the incidence angle leads to a significant shift in the defect mode, as well as of both the PBG edge's positions (from those in the case of normal incidence) to the higher frequency range. The analogous behavior of the PBG edges of the TE-mode is obtained in Ref. 28 for a 1D SC PC. Nevertheless the intensity behavior of the transmitted EMWs with the incidence angle increase differs drastically. For TE-polarization the intensity of the defect mode and both PBG edges decays steadily and becomes narrower with the increase in $\theta$, while for the TM-mode, first, the defect mode intensity grows slowly and broadens. Next, after reaching the maximal value at $\theta \approx 70^{\circ}$, the TMpolarized defect mode quickly drops in intensity and disappears practically, splitting into two vanishingly small branches at the grazing angle [Fig. 2(b)]. At the case of normal incidence $(\theta=0)$ the defect modes for both TE- and TMpolarizations are located in the center of the first PBG. In- creasing $\theta$ leaves this centered position of the TM-polarized defect mode intact practically for all values of $\theta$. The TEpolarized defect mode slightly deviates from the centered position at the grazing incidence angle [Fig. 2(a)].

The presence of the SC defect insert in the PC leads to modification of the transmittivity and reflectivity spectra of the PBGs as well as to appearance of absorption of the EMWs. In Figs. 3-5, we show the resulting transmittivity and absorptance spectra for the PCs with different values of $d_{s}$ equal to 10,20 , and $30 \mathrm{~nm}$, calculated for temperature $T$ $=77 \mathrm{~K}$.

It should be noted that the SC sublayer exhibits strongly pronounced optical anisotropy. Both diagonal components of the permittivity tensor $\epsilon_{s, x}(\omega)$ and $\epsilon_{s, y}(\omega)$ are complex, moreover, in the considered frequency range (first PGB) both real and imaginary parts of $\epsilon_{s, x}(\omega)$ are about two order of magnitude larger than the corresponding ones for $\epsilon_{s, y}(\omega)$. As $\epsilon_{s, y}(\omega)$ and $\epsilon_{s, x}(\omega)$ are responsible for the TE- and TMpolarized modes, respectively, it means that the decaying of the TM-polarized mode with the SC layer thickness $d_{s}$ inside the SC sublayer is much stronger than of the TE-polarized one. This difference in $\epsilon_{s, x}(\omega)$ and $\epsilon_{s, y}(\omega)$ leads to a drastic contrast in the behavior of TE- and TM-polarized modes with variation in the SC sublayer thickness $d_{s}$, as reported in our paper. $^{38}$

As was shown in Ref. 38, the variation in the SC sublayer thickness within some tens of nanometers does not produce visible changes in the PBG spectra of the $y$-polarized mode. The same behavior is typical to TE-polarized modes in the case of oblique incidence of the light. For this reason, we omit the corresponding figures of the PBG spectra for TE-modes for the PC with the SC sublayer $\left(d_{s}=10,20\right.$, and $30 \mathrm{~nm}$ ), referring to Fig. 2(a) above. In Figs. 3(a), 3(c), 4(a), 4(c), 5(a), and 5(c), we present the absorptance spectra for 

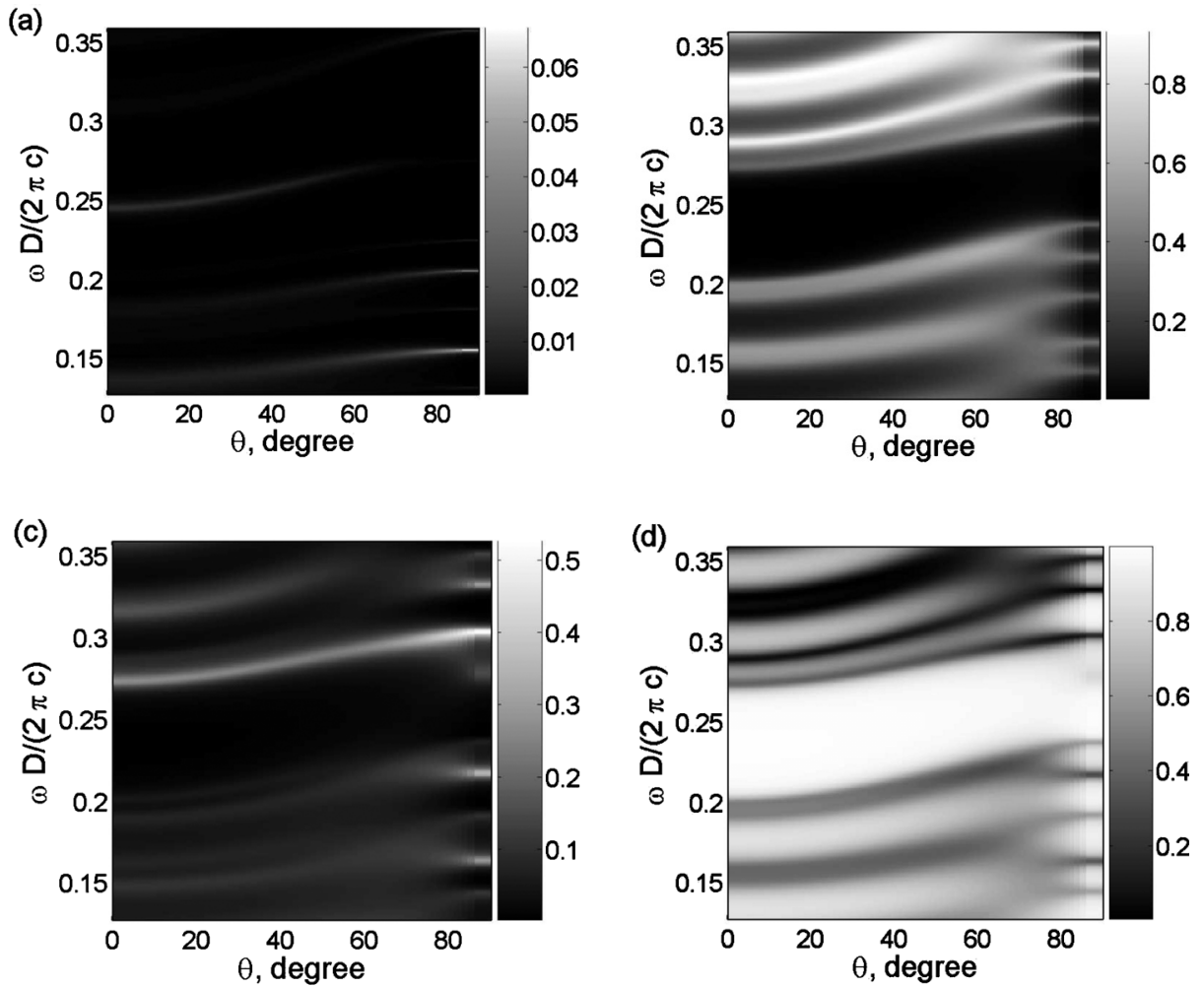

FIG. 4. The same as in Fig. 3 except for $d_{s}=20 \mathrm{~nm}$.

TE- and TM-modes. In Figs. 3(b), 3(d), 4(b), 4(d), 5(b), and $5(\mathrm{~d})$, we give the transmittivity and reflectivity spectra for TM-modes. Comparing the corresponding parts of Figs. 3-5, we can conclude that the increase in $d_{s}$ leads to a significant shift in the TM-polarized defect mode to the high frequency edge of the first PBG for all incidence angles. For the case of the PC with $d_{s}=10 \mathrm{~nm}$ [Fig. 4(b)] the defect mode is still separate from the PBG's edge, but at $d_{s}=20 \mathrm{~nm}$ it comes quite close to the edge, and at $d_{s}=30 \mathrm{~nm}$ it practically merges with it [Fig. 5(b)] for all incidence angles except $\theta$ $>70^{\circ}$.

In contrast to the case of a pure dielectric defect layer (Fig. 2), in Figs. 3-5 the TM-polarized defect modes are shifted from the PBG center toward the high frequency edge already at normal incidence $(\theta=0)$. This off-centered position remains with the variation in the incidence angle. The
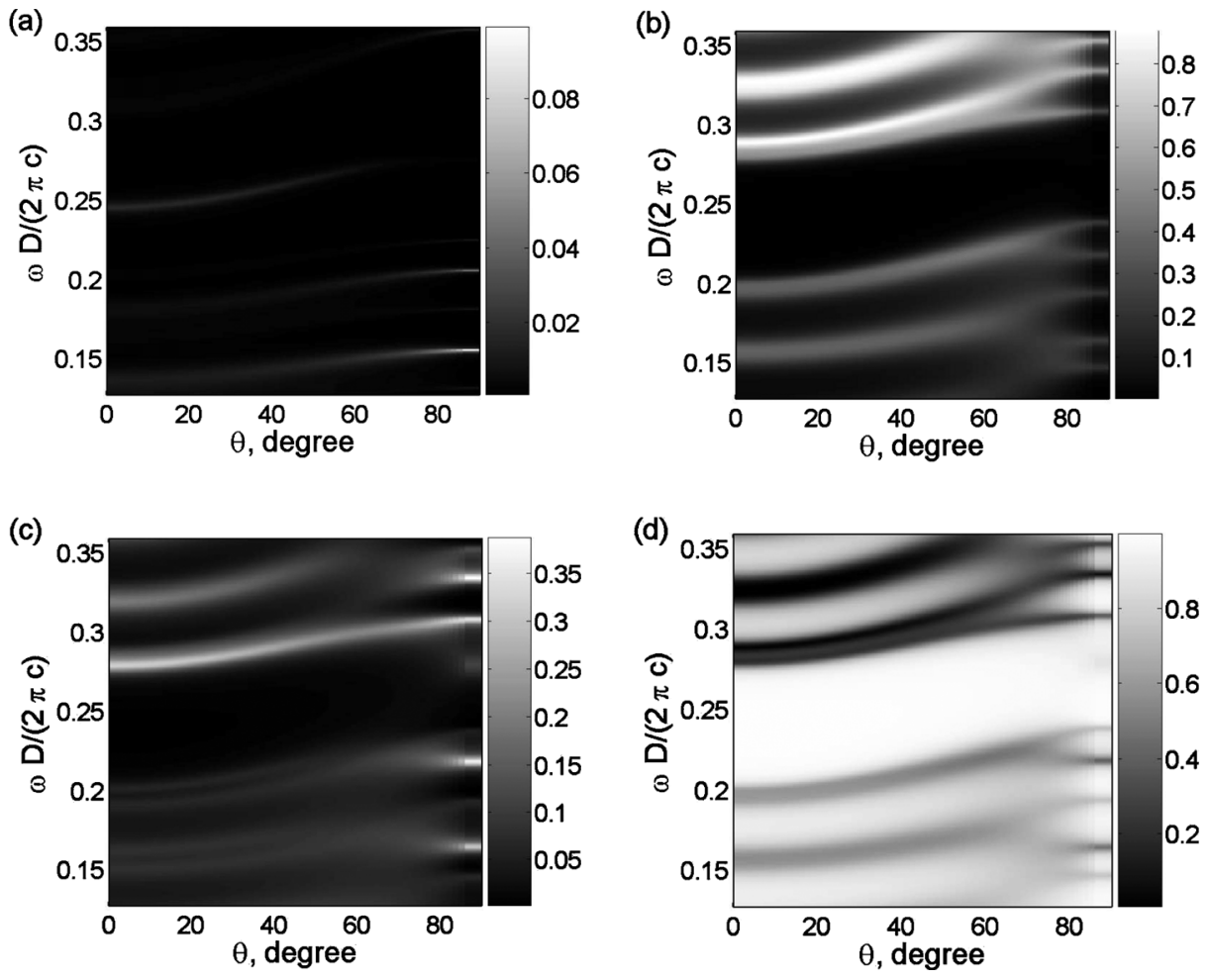

FIG. 5. The same as in Fig. 3 except for $d_{s}=30 \mathrm{~nm}$ 

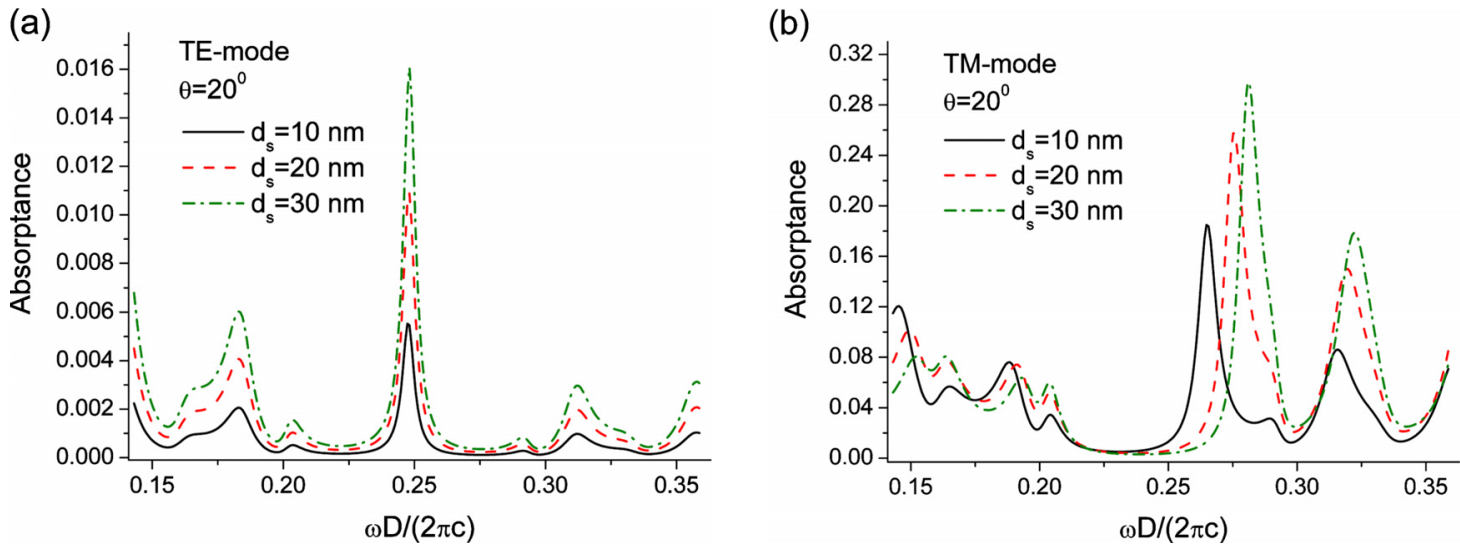

FIG. 6. (Color online) The absorptance profiles for the TE-modes (a) and TM-modes (b) in the cases of the light incidence at $\theta=20^{\circ}$, The solid, dashed, and dash-dotted lines correspond to the SC layer thicknesses of $d_{s}=10 \mathrm{~nm}, 20 \mathrm{~nm}$, and $30 \mathrm{~nm}$, respectively.

defect mode itself becomes wider and more intense with increasing of $\theta$ till approximately $70^{\circ}$. After that its intensity goes down to zero at $\theta=90^{\circ}$ without splitting, as in Fig. 2(b) for the PC with the pure dielectric defect layer. The same behavior of the TM-polarized defect mode at the grazing angle, we can see in Figs. 4(b) and 5(b).

In Figs. 3(a), 3(c), 4(a), 4(c), 5(a), and 5(c) we demonstrate the top views of the absorptance for TE-modes (a) and TM-modes (c) versus the normalized frequency and incidence angle for a set of values $d_{s}=10,20$, and $30 \mathrm{~nm}$. These figures are in direct correspondence with Figs. 3(b), 3(d), 4(b), 4(d), 5(b), and 5(d) for transmittivity and reflectivity, described above, as the energy conversation law requires $A^{(\gamma)}=1-\left(T^{(\gamma)}+R^{(\gamma)}\right)\left(A^{(\gamma)}, \gamma=\mathrm{TE}, \mathrm{TM}\right.$, is the absorptance of the corresponding mode). ${ }^{44}$ One can see, that the raised absorptance areas correspond to the defect modes and the edges of the PBGs. The difference in absorption features for the defect modes of TE- and TM-polarizations is obvious. The defect modes of TM-polarization are characterized by larger values of absorptance. For TE-polarized modes, the absorption decays with the incidence angle increase, while for TMpolarized mode it rises till essential values (about 0.5) at the grazing angles [for example, for the PC with $d_{s}=10 \mathrm{~nm}$ in Fig. 3(c)]. Besides the defect modes, the absorption is rising at the PBGs edges. For TE-modes there are narrow absorptance lines for large $\theta$ at the low frequency edges. For TMpolarized EMWs there is notable absorption on both PBG edges, especially for the cases of larger $d_{s}$ [see Figs. 4(c) and $5(\mathrm{c})]$.

Comparing the corresponding Figs. 3(a), 3(c), 4(a), 4(c), 5(a), and 5(c) one can see that the TM-mode absorptance exhibits essential changes with the increase in $d_{s}$, while the change in the TE-mode one is not so evident on the top view maps. Moreover, for all SC layer thicknesses, considered above, the TM-mode absorptance is much larger than the TE-mode one.

The common tendency in the modification of the absorptance spectra for TE-polarized defect modes for all considered thicknesses $d_{s}=10,20$, and $30 \mathrm{~nm}$ is a shift in the peaks, attributed to the defect mode, to the high frequency range with increasing of $\theta$. The value of the shift in the defect mode absorptance peak is quite large: 0.02878 in dimension- less units $\omega D /(2 \pi c)$ which corresponds to $10.84 \mathrm{THz}$ for $\theta$ going from normal incidence to $\theta=80^{\circ}$. Under this condition the magnitude of the absorptance peak undergoes almost a ten times reduction: from 0.0164 at normal incidence to a vanishingly small value 0.0018 for the case of $d_{s}=30 \mathrm{~nm}$.

In Figs. 6(a) and 6(b) we compare the positions and values of the absorption peaks for modes of TE- and TMpolarizations, respectively, for fixed incidence angle $\theta=20^{\circ}$. Figure 6(a) demonstrates that the positions of the absorption maximums remain steady with increase in the SC layer thickness. The corresponding results for TM-modes are given in Fig. 6(b). In contrast to Fig. 6(a), for TM-modes for all $\theta$ the absorptance value at the defect mode frequency dominates, exceeding the absorptance at the PBG edges and the nearest frequency ranges. Moreover, one can see that the positions of the absorptance maxima are shifted to higher frequency with increase in the SC layer thickness.

In Fig. 7, we present the dependence of the normalized PBG width $\Delta \omega / \Delta \omega(0)$ on the incidence angle $\theta$. Here $\Delta \omega(0)$ is the PBG width at $\theta=0$. The solid and short-dashed lines correspond to the PBG widths for TM- and TE-modes, respectively, for the case of the PC without a SC sublayer $\left(d_{s}=0\right)$. The dashed, dotted, and dash-dotted curves present TM-mode PBG widths for the PCs with the SC defect layers of thicknesses $d_{s}=10,20$, and $30 \mathrm{~nm}$, respectively.

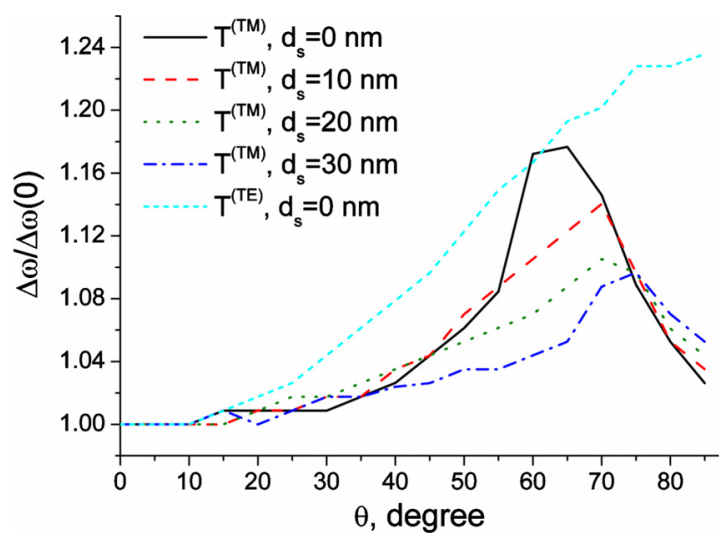

FIG. 7. (Color online) The normalized band width of the first PBG $\Delta \omega / \Delta \omega(0)$ as function of the incidence angle $\theta$ for TE- and TM-modes in the cases of the SC sublayer thicknesses $d_{s}=0,10,20$, and $30 \mathrm{~nm}$. 
The behavior of the PBG widths for TE- and TMpolarized modes is different. The thin SC sublayer of thickness of some tens of nanometers does not have a distinguishable effect on the PBG width for the TE-polarized mode, so the resulting PBG widths data for the PCs with $d_{s}=10,20$, and $30 \mathrm{~nm}$ produce practically identical outputs, presented by the same short-dashed curve on Fig. 7. For TM-modes, the presence of the maximum of the normalized PBG width $\Delta \omega / \Delta \omega(0)$ at some value of $\theta$ is a specific feature of its dependence on the incidence angle. The PBG width of the TE-polarized EMW exhibits no extremums and the broadening of the PBG with the increase in $\theta$ is more or less monotonic, achieving almost $24 \%$ of the value $\Delta \omega(0)$ at the grazing angle. As for TM-modes, the largest value of the PBG width is achieved for the PC with the pure dielectric defect layer (the solid curve) with the maximal PBG width equal to $18 \%$ of $\Delta \omega(0)$, attained in the vicinity of $\theta \approx 65^{\circ}$. The insert of the SC sublayer leads to a lowering of the maximum of the normalized PBG, accompanied by its shift to larger values of $\theta$. For $d_{s}=10 \mathrm{~nm}$ the maximal value of the PBG width is $14 \%$ of $\Delta \omega(0)$ at $\theta \approx 70^{\circ}$; for $d_{s}=20 \mathrm{~nm}$ it is $10 \%$ almost at the same incidence angle; and for $d_{s}=30 \mathrm{~nm}$ it is about $9 \%$ at $\theta \approx 75^{\circ}$.

\section{CONCLUSIONS}

In conclusion, we have investigated the $1 \mathrm{D}$ PC with a complex bilayer defect, composed of SC and dielectric constituents. The behavior of the defect modes with different polarizations as a function of the incidence angle variation is studied both analytically and numerically for different thicknesses of the SC sublayer. We showed that the intensity of the TE-polarized defect mode decreases with increasing incidence angle, whereas the intensity of the TM-polarized defect mode increases. The increase in the SC defect sublayer thickness leads to a shift in the defect mode of TMpolarization to higher frequencies up to its merging with the right PBG edge. The pronounced contrast in behavior of TEand TM-polarized modes is based on the in-plane anisotropy of dielectric tensor components of the SC sublayer.

\section{ACKNOWLEDGMENTS}

Useful discussions about the dielectric permittivity of superconductors with H. Rauh and Y. A. Genenko are gratefully acknowledged. This research is supported partly via Project FP7-PEOPLE-2009-IRSES No. 247556 "NoWaPhen" (N.N.D., A.E.Z., and I.L.L.), partly via NRF grant through q-Psi, Korea (Y.P.L.), and partly via grants from NWO - the Netherlands Organization for Scientific Research (Th.R.).

${ }^{1}$ J. D. Jannopoulos, S. G. Johnson, J. N. Winn, and R. D. Meade, Photonic Crystals: Molding the Flow of Light, 2nd ed. (Princeton University Press, Princeton, New York, 2008).

${ }^{2}$ K. Sakoda, Optical Properties of Photonic Crystals (Springer, Berlin, 2005)

${ }^{3}$ V. F. Shabanov, S. Y. Vetrov, and A. V. Shabanov, Optics of Real Photonic Crystals: Mesomorphic Defects and Inhomogeneities (Russian Academy of Sciences, Novosibirsk, 2005), in Russian.

${ }^{4}$ I. L. Lyubchanskii, N. N. Dadoenkova, M. I. Lyubchanskii, E. A. Shapovalov, and Th. Rasing, J. Phys. D 36, R277 (2003).

${ }^{5}$ M. Inoue, R. Fujikawa, A. Baryshev, A. Khanikaev, P. B. Lim, H. Ushida, O. Aktsipetrov, A. Fedyanin, T. Murzina, and A. Granovsky, J. Phys. D 39, R151 (2006).

${ }^{6}$ M. Levy and A. A. Jalali, J. Opt. Soc. Am. B 24, 1603 (2007).

${ }^{7}$ F. Wang and A. Lakhtakia, Appl. Phys. Lett. 92, 011115 (2008).

${ }^{8}$ A. Figotin and I. Vitebskiy, Waves Random Complex Media 20, 298 (2010).

${ }^{9}$ J. F. Scott, Ferroelectrics 293, 33 (2003).

${ }^{10}$ D. Scrymgeour, N. Malkova, S. Kim, and V. Gopalan, Appl. Phys. Lett. 82, 3176 (2003).

${ }^{11}$ S. Okamura, Y. Mochiduku, H. Motohara, and T. Shiosaki, Integr. Ferroelectr. 69, 303 (2005).

${ }^{12}$ T. V. Murzina, F. Y. Sychev, I. A. Kolmychek, and O. A. Aktsipetrov, Appl. Phys. Lett. 90, 161120 (2007).

${ }^{13}$ E. Mishina, A. Zaitsev, N. Ilyin, N. Sherstyuk, A. Sigov, Y. Golovko, V. Mukhortov, A. Kolesnikov, and Y. Lozovik, Appl. Phys. Lett. 91, 041107 (2007).

${ }^{14}$ Y. Fu, J. Zhang, X. Hu, and Q. Gong, J. Opt. 12, 075202 (2010).

${ }^{15}$ C. H. R. Ooi, T. C. Au Yeung, C. H. Kam, and T. K. Lim, Phys. Rev. B 61, 5920 (2000).

${ }^{16}$ Y. Chen, C. Zhang, Y. Zhu, S. Zhu, and N. Ming, Mater. Lett. 55, 12 (2002).

${ }^{17}$ S. Savel'ev, A. L. Rakhmanov, and F. Nori, Phys. Rev. Lett. 94, 157004 (2005).

${ }^{18}$ L. Feng, P. X. Liu, J. Ren, Y. F. Tang, Y. B. Chen, Y. F. Chen, and Y. Y. Zhu, J. Appl. Phys. 97, 073104 (2005).

${ }^{19}$ A. Pimenov, A. Loidl, P. Przyslupski, and B. Dabrovski, Phys. Rev. Lett. 95, 247009 (2005).

${ }^{20}$ A. H. Aly, J. Supercond. Novel Magn. 21, 421 (2008).

${ }^{21}$ A. H. Aly, S.-W. Ryu, H.-T. Hsu, and C.-J. Wu, Mater. Chem. Phys. 113, 382 (2009)

${ }^{22}$ W. M. Lee, P. M. Hui, and D. Stroud, Phys. Rev. B 51, 8634 (1995).

${ }^{23}$ H. Takeda and K. Yoshino, Phys. Rev. B 67, 245109 (2003).

${ }^{24}$ H. Takeda, K. Yoshino, and A. A. Zakhidov, Phys. Rev. B 70, 085109 (2004).

${ }^{25}$ O. L. Berman, Y. E. Lozovik, S. L. Eiderman, and R. D. Coalson, Phys. Rev. B 74, 092505 (2006).

${ }^{26}$ A. N. Poddubny, E. L. Ivchenko, and Y. E. Lozovik, Solid State Commun. 146, 143 (2008).

${ }^{27}$ C. Cheng, C. Xu, T. Zhou, X.-F. Zhang, and Y. Xu, J. Phys.: Condens. Matter 20, 275203 (2008).

${ }^{28}$ C.-J. Wu, C.-L. Lui, and T.-J. Yang, J. Opt. Soc. Am. B 26, 2089 (2009).

${ }^{29}$ C. H. R. Ooi and C. H. Kam, J. Opt. Soc. Am. B 27, 458 (2010).

${ }^{30}$ H.-M. Lee, C.-Y. Lin, L. Horng, and J.-C. Wu, J. Appl. Phys. 107, 09E119 (2010).

${ }^{31}$ H.-M. Lee and J.-C. Wu, J. Appl. Phys. 107, 09E149 (2010)

${ }^{32}$ K. B. Thapa, S. Srivastava, and S. Tiwari, J. Supercond. Novel Magn. 23, 517 (2010).

${ }^{33}$ C.-J. Wu, C.-L. Lui, and W.-K. Kuo, J. Electromagn. Waves Appl. 23, 1113 (2009).

${ }^{34}$ E. Di Gennaro, C. Zannini, S. Savo, A. Andreone, M. R. Masullo, G. Castaldi, I. Gallina, and V. Galdi, New J. Phys. 11, 113022 (2009).

${ }^{35}$ H.-F. Huang, J.-F. Mao, X.-C. Li, and Z. Li, IEEE Trans. Appl. Supercond. 15, 3827 (2005).

${ }^{36}$ T.-H. Pei and Y.-T. Huang, J. Appl. Phys. 101, 084502 (2007).

${ }^{37}$ H. Rauh and Y. A. Genenko, J. Phys.: Condens. Matter 20, 145203 (2008).

${ }^{38}$ I. L. Lyubchanskii, N. N. Dadoenkova, A. E. Zabolotin, Y. P. Lee, and Th. Rasing, J. Opt. A, Pure Appl. Opt. 11, 114014 (2009).

${ }^{39}$ D. W. Berreman, J. Opt. Soc. Am. 62, 502 (1972).

${ }^{40}$ P. Yeh, Surf. Sci. 96, 41 (1980).

${ }^{41}$ P. Yeh, Optical Waves in Layered Media (Wiley, New York, 1988).

${ }^{42}$ I. L. Lyubchanskii, N. N. Dadoenkova, M. I. Lyubchanskii, E. A. Shapovalov, A. Lakhtakia, and Th. Rasing, Appl. Phys. Lett. 85, 5932 (2004).

${ }^{43}$ E. D. Palik, Handbook of Optical Constants of Solids (Academic, New York, 1991).

${ }^{44} \mathrm{M}$. Born and E. Wolf, Principles of Optics, 7th ed. (Cambridge University Press, Cambridge, 1999). 